\title{
Analysis of the Structured Perturbation for the BCSCB Linear System
}

\author{
Xia Tang ${ }^{1,2}$ and Zhaolin Jiang ${ }^{1}$ \\ ${ }^{1}$ Department of Mathematics, Linyi University, Linyi, Shandong 276000, China \\ ${ }^{2}$ Department of Mathematics, Shandong Normal University, Jinan, Shandong 250014, China \\ Correspondence should be addressed to Zhaolin Jiang; jzh1208@sina.com
}

Received 22 July 2014; Accepted 10 September 2014

Academic Editor: Zidong Wang

Copyright (c) $2015 \mathrm{X}$. Tang and Z. Jiang. This is an open access article distributed under the Creative Commons Attribution License, which permits unrestricted use, distribution, and reproduction in any medium, provided the original work is properly cited.

\begin{abstract}
Circulant and block circulant type matrices are important tools in solving networked systems. In this paper, based on the style spectral decomposition of the basic circulant matrix and the basic skew circulant matrix, the block style spectral decomposition of the BCSCB matrix is obtained. And then, the structure perturbation is analysed, which includes the condition number and relative error of the BCSCB linear system. Then the optimal backward perturbation bound of the BCSCB linear system is discussed. Simultaneously, the algorithm for the optimal backward perturbation bound is given. Finally, a numerical example is provided to verify the effectiveness of the algorithm.
\end{abstract}

\section{Introduction}

It is an active objective that circulant and block circulant type matrices are applied to networks engineering. The stability region in the parameters space is extended by the breaking of a delayed ring neural network where the form of time-delay systems is $\dot{x}+A x(t)+B x(t-\tau)=0$, where $B$ is a circulant matrix, if the number of the neurons is sufficiently large in [1]. In [2], the question of when circulant quantum spin networks with nearest-neighbor couplings can give perfect state transfer is solved. The properties of linear diffusion algorithm are investigated both by a worst-case analysis and by a probabilistic analysis and are shown to depend on the spectral properties of the circulant matrix in [3]. A viable option for increasing the lifetime of the sensor network for a small loss in accuracy of the query results whose matrices are circulant is offered in [4]. In [5], the authors considered the kinetics of an autocatalytic reaction network in which replication and catalytic actions are separated by a translation step. They found that the behavior of such a system is closely related to second-order replicator equations, where the second-order replicator equations are circulant interaction matrices. In order to obtain the optimal routing in double loop networks, the problem of finding the shortest path in circulant graphs with an arbitrary number of jumps is studied in [6].

A block circulant with skew circulant blocks matrix with the first row $\left(c_{11}, \ldots, c_{1 m}, c_{21}, \ldots, c_{2 m}, \ldots, c_{n 1}, \ldots, c_{n m}\right)$ has the following form:

$$
\mathbb{C}=\left(\begin{array}{ccccc}
C_{1} & C_{2} & \cdots & C_{n-1} & C_{n} \\
C_{n} & C_{1} & C_{2} & \cdots & C_{n-1} \\
\vdots & \ddots & \ddots & \ddots & \vdots \\
C_{3} & \ddots & C_{n} & C_{1} & C_{2} \\
C_{2} & C_{3} & \cdots & C_{n} & C_{1}
\end{array}\right),
$$

and for any $k=1,2, \ldots, n$,

$$
C_{k}=\left(\begin{array}{ccccc}
c_{k 1} & c_{k 2} & \cdots & c_{k(m-1)} & c_{k m} \\
-c_{k m} & c_{k 1} & c_{k 2} & \cdots & c_{k(m-1)} \\
\vdots & \ddots & \ddots & \ddots & \vdots \\
-c_{k 3} & \ddots & -c_{k m} & c_{k 1} & c_{k 2} \\
-c_{k 2} & -c_{k 3} & \cdots & -c_{k m} & c_{k 1}
\end{array}\right)
$$

The matrix $\mathbb{C}$ is denoted by $\operatorname{BCSCB}\left(c_{11}, \ldots, c_{1 m}, \ldots, c_{n 1}, \ldots\right.$, $\left.c_{n m}\right)$. 
Rigal and Gaches [7] considered a posteriori analysis of the compatibility of a computed solution to the uncertain data of a linear system by some new theorems generalizing a result of Oettli and Prager. In [8], the style spectral decomposition of the skew circulant matrix is given and the optimal backward perturbation analysis for the skew circulant linear system is discussed. Liu and Guo [9] obtained the bound of the optimal backward perturbation for a block circulant linear system. J.-G. Sun and Z. Sun [10] studied the optimal backward perturbation bounds for undetermined systems. In [11], the optimal backward perturbation analysis for the block skew circulant linear system with skew circulant blocks is given by Li et al.

\section{The Block Style Spectral Decomposition of the BCSCB Matrix}

Let matrix $\mathbb{C}$ be a BCSCB matrix as the form (1); then by using the properties of Kronecker products in [12], the $\mathbb{C}$ can be decomposed as

$$
\mathbb{C}=\sum_{k=1}^{n}\left(\Pi^{k-1} \otimes C_{k}\right)
$$

where $\Pi$ is a square matrix of order $n$, and it has the following form:

$$
\Pi=\left(\begin{array}{ccccc}
0 & 1 & 0 & \cdots & 0 \\
0 & 0 & 1 & \cdots & 0 \\
\vdots & \ddots & \ddots & \ddots & \vdots \\
0 & \cdots & 0 & 0 & 1 \\
1 & 0 & \cdots & 0 & 0
\end{array}\right)
$$

Based on (2.5) and (2.6) in [9], the style spectral decomposition of the matrix $\Pi$ is

$$
\Pi=Q \Pi_{0} Q^{T},
$$

where $Q$ is an orthogonal matrix.

When $n$ is even,

$$
\begin{gathered}
\Pi_{0}=\left(\begin{array}{llll}
\pi_{1} & & & \\
& \pi_{2} & & \\
& & \ddots & \\
& & & \pi_{n / 2}
\end{array}\right), \\
\pi_{n / 2}=\left(\begin{array}{cc}
-1 & 0 \\
0 & 1
\end{array}\right), \quad \pi_{j}=\left(\begin{array}{cc}
\cos \theta_{j} & \sin \theta_{j} \\
-\sin \theta_{j} & \cos \theta_{j}
\end{array}\right), \\
\theta_{j}=\frac{2 j}{n} \pi, \quad j=1,2, \ldots, \frac{n}{2}-1 .
\end{gathered}
$$

When $n$ is odd,

$$
\Pi_{0}=\left(\begin{array}{ccccc}
\pi_{1} & & & & \\
& \pi_{2} & & & \\
& & \ddots & & \\
& & & \pi_{(n-1) / 2} & 1
\end{array}\right),
$$

$$
\begin{array}{r}
\pi_{j}=\left(\begin{array}{cc}
\cos \theta_{j} & \sin \theta_{j} \\
-\sin \theta_{j} & \cos \theta_{j}
\end{array}\right), \quad \theta_{j}=\frac{2 j}{n} \pi, \\
j=1,2, \ldots, \frac{n-1}{2} .
\end{array}
$$

Taking (3) and (5) into consideration, the matrix $\mathbb{C}$ can be decomposed as

$$
\begin{aligned}
\mathbb{C} & =\sum_{k=1}^{n}\left(\Pi^{k-1} \otimes C_{k}\right) \\
& =\sum_{k=1}^{n}\left(Q \Pi_{0}^{k-1} Q^{T}\right) \otimes C_{k} \\
& =\sum_{k=1}^{n}\left(Q \otimes I_{m}\right)\left(\Pi_{0}^{k-1} \otimes C_{k}\right)\left(Q^{T} \otimes I_{m}\right) \\
& =\left(Q \otimes I_{m}\right) \sum_{k=1}^{n}\left(\Pi_{0}^{k-1} \otimes C_{k}\right)\left(Q^{T} \otimes I_{m}\right) .
\end{aligned}
$$

$Q \otimes I_{m}$ is an orthogonal matrix obviously. So (8) is the block style spectral decomposition of the matrix $\mathbb{C}$.

\section{Analysis of the Structured Perturbation}

The structured perturbation analysis for BCSCB linear system is given in this section. We discuss the condition number and the relative error of the BCSCB linear system. The optimal backward perturbation bound of the BCSCB linear system is analysed. And, at the end of the section, we give the algorithm for the optimal backward perturbation bound.

3.1. Condition Number and Relative Error of BCSCB Linear System. Consider

$$
\mathbb{C} x=b,
$$

where $\mathbb{C}$ is defined in (1).

From (8) and the property of Kronecker products in [12], the matrix $\mathbb{C}$ can be expressed by using the elements in its first row as

$$
\begin{aligned}
\mathbb{C} & =\sum_{k=1}^{n}\left(\Pi^{k-1} \otimes C_{k}\right) \\
& =\sum_{k=1}^{n}\left[\Pi^{k-1} \otimes\left(\sum_{l=1}^{m} c_{k l} \Psi^{l-1}\right)\right] \\
& =\sum_{k=1}^{n} \sum_{l=1}^{m} c_{k l}\left(\Pi^{k-1} \otimes \Psi^{l-1}\right),
\end{aligned}
$$

where $\Psi$ is a square matrix of order $m$, and it has the following form:

$$
\Psi=\left(\begin{array}{ccccc}
0 & 1 & 0 & \cdots & 0 \\
0 & 0 & 1 & \cdots & 0 \\
\vdots & \ddots & \ddots & \ddots & \vdots \\
0 & \cdots & 0 & 0 & 1 \\
-1 & 0 & \cdots & 0 & 0
\end{array}\right)
$$


Based on (10) and (11) in [8], the style spectral decomposition of the matrix $\Psi$ is

$$
\Psi=J \Psi_{0} J^{T}
$$

where $J$ is an orthogonal matrix.

When $m$ is even,

$$
\Psi_{0}=\left(\begin{array}{llll}
\psi_{1} & & & \\
& \psi_{2} & & \\
& & \ddots & \\
& & & \psi_{m / 2}
\end{array}\right)
$$

When $m$ is odd,

$$
\begin{aligned}
& \Psi_{0}=\left(\begin{array}{lllll}
\psi_{1} & & & & \\
& \psi_{2} & & & \\
& & \ddots & & \\
& & & \psi_{(m-1) / 2} & -1
\end{array}\right) \text {, } \\
& \psi_{h}=\left(\begin{array}{cc}
\cos \theta_{h} & \sin \theta_{h} \\
-\sin \theta_{h} & \cos \theta_{h}
\end{array}\right), \quad \theta_{h}=\frac{2 h-1}{n} \pi, \\
& h= \begin{cases}1,2, \ldots, \frac{m}{2}, & m \text { is even. } \\
1,2, \ldots, \frac{m-1}{2}, & m \text { is odd }\end{cases}
\end{aligned}
$$

Furthermore, (10) can be expressed as follows:

$$
\mathbb{C}=\mathbb{Q}\left(\sum_{k=1}^{n} \sum_{l=1}^{m} c_{k l} \Pi_{0}^{k-1} \otimes \Psi_{0}^{l-1}\right) \mathbb{Q}^{T}
$$

and here $\mathbb{Q}=\left(Q \otimes I_{m}\right)\left(I_{n} \otimes J\right)$, where $I_{n}$ and $I_{m}$ are identity matrices with orders $n$ and $m$, respectively.

The problem will be discussed at two different situations.

(1) When $n$ is even,

$$
\begin{aligned}
& \sum_{k=1}^{n} \sum_{l=1}^{m} c_{k l} \Pi_{0}^{k-1} \otimes \Psi_{0}^{l-1}=\left(\begin{array}{cccc}
\Lambda_{11} & & & \\
& \ddots & & \\
& & \Lambda_{t t} & \\
& & & \Upsilon_{1}
\end{array}\right) \\
& \Lambda_{p p}=\sum_{k=1}^{n} \sum_{l=1}^{m} c_{k l} \pi_{p}^{k-1} \otimes \Psi_{0}^{l-1}, \\
& t=\frac{n}{2}-1, \quad p=1,2, \ldots, t, \\
& \Upsilon_{1}=\sum_{k=1}^{n} \sum_{l=1}^{m} c_{k l} \operatorname{diag}\left(-\Psi_{0}^{l-1}, \Psi_{0}^{l-1}\right) \text {. }
\end{aligned}
$$

(2) When $n$ is odd,

$$
\sum_{k=1}^{n} \sum_{l=1}^{m} c_{k l} \Pi_{0}^{k-1} \otimes \Psi_{0}^{l-1}=\left(\begin{array}{cccc}
\Lambda_{11} & & & \\
& \ddots & & \\
& & \Lambda_{t t} & \\
& & & \Upsilon_{2}
\end{array}\right)
$$

$$
\begin{aligned}
& \Lambda_{p p}=\sum_{k=1}^{n} \sum_{l=1}^{m} c_{k l} \pi_{p}^{k-1} \otimes \Psi_{0}^{l-1}, \\
& t=\frac{n-1}{2}, \quad p=1,2, \ldots, t, \\
& \Upsilon_{2}=\sum_{k=1}^{n} \sum_{l=1}^{m} c_{k l} \Psi_{0}^{l-1} .
\end{aligned}
$$

We denote by $\omega_{i}(i=1,2, \ldots, n)$ the eigenvalues of matrix $\Pi[9]$, and $\delta_{j}(j=1,2, \ldots, m)$ are denoted as the eigenvalues of matrix $\Psi$ [8], and then the eigenvalues of $\mathbb{C}$ are obtained (refer to $[12,13])$. Consider

$$
\lambda_{i j}=\sum_{k=1}^{n} \sum_{l=1}^{m} c_{k l} \omega_{i}^{k-1} \delta_{j}^{l-1} .
$$

Lemma 1. $\mathbb{C}$ is a nonsingular matrix if and only if $f\left(\omega_{i}, \delta_{j}\right) \neq$ $0(i=1,2, \ldots, n, j=1,2, \ldots, m)$, where

$$
f\left(\omega_{i}, \delta_{j}\right)=\lambda_{i j}=\sum_{k=1}^{n} \sum_{l=1}^{m} c_{k l} \omega_{i}^{k-1} \delta_{j}^{l-1} .
$$

Let

$$
\begin{gathered}
\sigma_{i j}=\left|f\left(\omega_{i}, \delta_{j}\right)\right|, \quad i=1,2, \ldots, n, j=1,2, \ldots, m, \\
\kappa=\frac{\max \left\{\sigma_{i j}\right\}}{\min \left\{\sigma_{i j}\right\}} .
\end{gathered}
$$

Theorem 2. If $\mathbb{C}=\operatorname{BCSCB}\left(c_{11}, \ldots, c_{1 m}, \ldots, c_{n 1}, \ldots, c_{n m}\right)$, then the singular values of the matrix $\mathbb{C}$ are $\sigma_{11}, \ldots, \sigma_{1 m}, \sigma_{21}, \ldots$, $\sigma_{2 m}, \ldots, \sigma_{n 1}, \ldots, \sigma_{n m}$.

Proof. Assume the conjugate transpose of $\mathbb{C}$ is

$$
\mathbb{C}^{*}=\left(\begin{array}{ccccc}
C_{1}^{*} & C_{n}^{*} & \cdots & C_{3}^{*} & C_{2}^{*} \\
C_{2}^{*} & C_{1}^{*} & \cdots & \vdots & C_{3}^{*} \\
\vdots & C_{2}^{*} & \cdots & C_{n}^{*} & \vdots \\
C_{n-1}^{*} & \vdots & \ddots & C_{1}^{*} & C_{n}^{*} \\
C_{n}^{*} & C_{n-1}^{*} & \cdots & C_{2}^{*} & C_{1}^{*}
\end{array}\right) .
$$

By a direct calculation, $\mathbb{C}$ is a normal matrix as $\mathbb{C} \mathbb{C}^{*}=$ $\mathbb{C}^{*} \mathbb{C}$. Then matrix $\mathbb{C}$ is a unitarily diagonalizable matrix based on Theorem 2.5.4 in [14]. Then there exists a unitary matrix $\mathbb{U} \in M_{m n}$, such that

$$
\mathbb{U}^{*} \mathbb{C} \mathbb{U}=\Lambda=\operatorname{diag}\left(\lambda_{11}, \ldots, \lambda_{1 m}, \ldots, \lambda_{n 1}, \ldots, \lambda_{n m}\right),
$$

where $\lambda_{i j}(i=1,2, \ldots, n, j=1,2, \ldots, m)$ are the eigenvalues of matrix $\mathbb{C}$. Taking the conjugate transpose at both sides of (22), we get

$$
\mathbb{U}^{*} \mathbb{C}^{*} \mathbb{U}=\Lambda=\operatorname{diag}\left(\bar{\lambda}_{11}, \ldots, \bar{\lambda}_{1 m}, \ldots, \bar{\lambda}_{n 1}, \ldots, \bar{\lambda}_{n m}\right) ;
$$


then

$$
\begin{aligned}
\mathbb{U}^{*}\left(\mathbb{C}^{*} \mathbb{C}\right) \mathbb{U} \\
\quad=\left(\mathbb{U}^{*} \mathbb{C}^{*} \mathbb{U}\right)\left(\mathbb{U}^{*} \mathbb{C} \mathbb{U}\right) \\
\quad=\operatorname{diag}\left(\left|\lambda_{11}\right|^{2}, \ldots,\left|\lambda_{1 m}\right|^{2}, \ldots,\left|\lambda_{n 1}\right|^{2}, \ldots,\left|\lambda_{n m}\right|^{2}\right) .
\end{aligned}
$$

And $\left|\lambda_{i j}\right|^{2}$ are the eigenvalues of the matrix $\mathbb{C}^{*} \mathbb{C}$, for any $i=1,2, \ldots, n, j=1,2, \ldots, m$. Therefore, the singular values of $\mathbb{C}$ are

$$
\sigma_{i j}(\mathbb{C})=\left[\lambda_{i j}\left(\mathbb{C}^{*} \mathbb{C}\right)\right]^{1 / 2}=\left|\lambda_{i j}\right|
$$

Recall (19) and (20); the proof is completed.

As the definition of the spectral norm of matrix $\mathbb{C}$ is

$$
\|\mathbb{C}\|_{2}=\max _{\substack{1 \leq i \leq n \\ 1 \leq j \leq m}}\left[\lambda_{i j}\left(\mathbb{C}^{*} \mathbb{C}\right)\right]^{1 / 2}
$$

via Theorem 2, the following corollary is obtained.

Corollary 3. Let $\mathbb{C}=\operatorname{BCSCB}\left(c_{11}, \ldots, c_{1 m}, \ldots, c_{n 1}, \ldots, c_{n m}\right)$; then the spectrum norm of matrix $\mathbb{C}$ is

$$
\|\mathbb{C}\|_{2}=\max _{\substack{1 \leq i \leq n \\ 1 \leq j \leq m}}\left\{\sigma_{i j}\right\} .
$$

Let $\Delta \mathbb{C}$ be a perturbation of the coefficient matrix $\mathbb{C}$ and let $\Delta b$ be a perturbation of the vector $b$, where $\Delta \mathbb{C}=$ $\operatorname{BCSCB}\left(\varepsilon c_{11}, \ldots, \varepsilon c_{1 m}, \ldots, \varepsilon c_{n 1}, \ldots, \varepsilon c_{n m}\right)$ has the following form:

$$
\Delta \mathbb{C}=\left(\begin{array}{cccc}
\Delta C_{1} & \cdots & \Delta C_{n-1} & \Delta C_{n} \\
\Delta C_{n} & \Delta C_{1} & \cdots & \Delta C_{n-1} \\
\vdots & \ddots & \ddots & \vdots \\
\Delta C_{2} & \cdots & \Delta C_{n} & \Delta C_{1}
\end{array}\right)
$$

and for any $k=1,2, \ldots, m$,

$$
\Delta C_{k}=\left(\begin{array}{cccc}
\varepsilon c_{k 1} & \cdots & \varepsilon_{k(m-1)} & \varepsilon c_{k m} \\
-\varepsilon c_{k m} & \varepsilon c_{k 1} & \cdots & \varepsilon c_{k(m-1)} \\
\vdots & \ddots & \ddots & \vdots \\
-\varepsilon c_{k 2} & \cdots & -\varepsilon c_{k m} & \varepsilon c_{k 1}
\end{array}\right)
$$

Let

$$
\begin{gathered}
\widehat{\mathbb{C}}=\mathbb{C}+\Delta \mathbb{C}, \quad \widehat{b}=b+\Delta b, \quad \Delta b=\varepsilon b, \\
\widehat{f}\left(\omega_{i}, \delta_{j}\right)=\sum_{k=1}^{n} \sum_{l=1}^{m}\left(c_{k l}+\varepsilon c_{k l}\right) \omega_{i}^{k-1} \delta_{j}^{l-1} .
\end{gathered}
$$

If

$$
\sum_{k=1}^{n} \sum_{l=1}^{m}\left|\varepsilon c_{k l}\right|<\min _{\substack{1 \leq i \leq n \\ 1 \leq j \leq m}}\left\{\sigma_{i j}\right\}
$$

then

$$
\begin{aligned}
&\left|\widehat{f}\left(\omega_{i}, \delta_{j}\right)\right|=\left|\sum_{k=1}^{n} \sum_{l=1}^{m}\left(c_{k l}+\varepsilon c_{k l}\right) \omega_{i}^{k-1} \delta_{j}^{l-1}\right| \\
& \geq\left|\sum_{k=1}^{n} \sum_{l=1}^{m} c_{k l} \omega_{i}^{k-1} \delta_{j}^{l-1}\right| \\
&-\sum_{k=1}^{n} \sum_{l=1}^{m}\left|\varepsilon c_{k l}\right|\left|\omega_{i}\right|^{k-1}\left|\delta_{j}\right|^{l-1} \\
& \geq \min _{\substack{1 \leq i \leq n \\
1 \leq j \leq m}}\left\{\sigma_{i j}\right\}-\sum_{k=1}^{n} \sum^{m}\left|\varepsilon c_{k l}\right|>0 ;
\end{aligned}
$$

through Lemma $1, \widehat{\mathbb{C}}$ is a nonsingular matrix. Let

$$
\sigma_{\min }=\min _{\substack{1 \leq i \leq n \\ 1 \leq j \leq m}}\left\{\sigma_{i j}\right\}, \quad \rho=\sum_{k=1}^{n} \sum_{l=1}^{m}\left|\varepsilon c_{k l}\right| .
$$

By $\mathbb{C} x=b, \widehat{\mathbb{C}} \widehat{x}=\widehat{b}$, we obtain

$$
\begin{aligned}
& \widehat{x}-x=\widehat{\mathbb{C}}^{-1} \widehat{b}-\mathbb{C}^{-1} b \\
& =\widehat{\mathbb{C}}^{-1}(b+\varepsilon b)-\mathbb{C}^{-1} b \\
& =\widehat{\mathbb{C}}^{-1} \varepsilon b+\left(\widehat{\mathbb{C}}^{-1}-\mathbb{C}^{-1}\right) b \\
& =\widehat{\mathbb{C}}^{-1} \varepsilon b+\left(\widehat{\mathbb{C}}^{-1}-\mathbb{C}^{-1}\right) \mathbb{C} x \\
& =\widehat{\mathbb{C}}^{-1} \varepsilon b+\widehat{\mathbb{C}}^{-1}(\mathbb{C}-\widehat{\mathbb{C}}) x, \\
& \|\widehat{x}-x\|_{2} \leq\left\|\widehat{\mathbb{C}}^{-1}\right\|_{2}\|\varepsilon b\|_{2}+\left\|\widehat{\mathbb{C}}^{-1}\right\|_{2}\|\widehat{\mathbb{C}}-\mathbb{C}\|_{2}\|x\|_{2} \\
& \leq \frac{\|\varepsilon b\|_{2}}{\sigma_{\min }-\rho}+\frac{\|\widehat{\mathbb{C}}-\mathbb{C}\|_{2}\|x\|_{2}}{\sigma_{\min }-\rho}, \\
& \frac{\|\widehat{x}-x\|_{2}}{\|x\|_{2}} \leq \frac{\|\varepsilon b\|_{2}}{\left(\sigma_{\min }-\rho\right)\|x\|_{2}}+\frac{\|\widehat{\mathbb{C}}-\mathbb{C}\|_{2}}{\sigma_{\min }-\rho} \\
& =\frac{\|\mathbb{C}\|_{2}}{\sigma_{\min }-\rho}\left[\frac{\|\varepsilon b\|_{2}}{\|\mathbb{C}\|_{2}\|x\|_{2}}+\frac{\|\widehat{\mathbb{C}}-\mathbb{C}\|_{2}}{\|\mathbb{C}\|_{2}}\right] \\
& \leq \frac{\|\mathbb{C}\|_{2}}{\sigma_{\min }-\rho}\left[\frac{\|\varepsilon b\|_{2}}{\|b\|_{2}}+\frac{\|\widehat{\mathbb{C}}-\mathbb{C}\|_{2}}{\|\mathbb{C}\|_{2}}\right] \text {, }
\end{aligned}
$$

where

$$
\|\mathbb{C}\|_{2}=\max _{\substack{1 \leq i \leq n \\ 1 \leq j \leq m}}\left\{\sigma_{i j}\right\}
$$


$\widehat{\mathbb{C}}-\mathbb{C}=\Delta \mathbb{C}$ is a BCSCB matrix apparently, and $\|\mathbb{C}-\widehat{\mathbb{C}}\|_{2}=$ $|-1|\|\widehat{\mathbb{C}}-\mathbb{C}\|_{2}=\|\widehat{\mathbb{C}}-\mathbb{C}\|_{2}$. So

$$
\begin{aligned}
\|\widehat{\mathbb{C}}-\mathbb{C}\|_{2} & =\max _{1 \leq j \leq n}\left|\sum_{k=1 l=1}^{n} \sum_{1 \leq j}^{m} \varepsilon c_{k l} \omega_{i}^{k-1} \delta_{j}^{l-1}\right| \\
& \leq \max _{1 \leq i \leq n} \sum_{\substack{1 \leq j \leq m \\
k=1 l=1}}^{m}\left|\varepsilon c_{k l}\right|\left|\omega_{i}\right|^{k-1}\left|\delta_{j}\right|^{l-1} \\
& =\sum_{k=1 l=1}^{n} \sum_{l=1}^{m}\left|\varepsilon c_{k l}\right|=\rho .
\end{aligned}
$$

The following theorem can be obtained.

Theorem 4. Let $\mathbb{C}, \widehat{\mathbb{C}}, \Delta b, \rho$, and $\sigma_{\min }$ be defined as above. If $\rho<\sigma_{\min }$, then

$$
\frac{\|\hat{x}-x\|_{2}}{\|x\|_{2}} \leq \frac{\sigma_{\max }}{\sigma_{\min }-\rho}\left(\frac{\|\varepsilon b\|_{2}}{\|b\|_{2}}+\frac{\rho}{\sigma_{\max }}\right),
$$

where

$$
\sigma_{\max }=\|\mathbb{C}\|_{2} .
$$

Remark 5. The condition number $\kappa$ of the BCSCB matrix can be easily computed with the basis of (37) and (38), the same as the bound of perturbation (37).

\subsection{Optimal Backward Perturbation Bound of the BCSCB} Linear System. In this part, a new method is given to obtain the minimal value of the perturbation bound, which is only related to the perturbation of the coefficient matrix and the vector. At the end of this part, the algorithm for the optimal backward perturbation bound is given.

Let $\widehat{x}$ be an approximate solution to $\mathbb{C} x=b$ and let

$$
\begin{gathered}
\Omega \equiv\{(\Delta \mathbb{C}, \Delta b) \mid(\mathbb{C}+\Delta \mathbb{C}) \hat{x}=b+\Delta b\}, \\
\xi(\widehat{x}) \equiv \inf _{(\Delta \mathbb{C}, \Delta b) \in \Omega}\|\Delta \mathbb{C}, \Delta b\|, \\
(\mathbb{C}+\Delta \mathbb{C}) \widehat{x}=b+\Delta b,
\end{gathered}
$$

which is equal to

$$
(\Delta \mathbb{C}, \Delta b)\left(\begin{array}{c}
\widehat{x} \\
-1
\end{array}\right)=b-\mathbb{C} \hat{x} .
$$

According to [7], we can get

$$
\xi(\widehat{x})=\frac{\|b-\mathbb{C} \widehat{x}\|_{2}}{\sqrt{1+\|\widehat{x}\|_{2}^{2}}}
$$

$(\|\cdot\|$ is unitary invariant norm)

Let $\widehat{x}$ be an approximate solution to $\mathbb{C} x=b$, where $\mathbb{C}$ is defined in (1):

$$
\Omega \equiv\{(\Delta \mathbb{C}, \Delta b) \mid(\mathbb{C}+\Delta \mathbb{C}) \hat{x}=b+\Delta b,
$$

$\Delta \mathbb{C}$ is a BCSCB matrix,

$$
\xi(\widehat{x}) \equiv \inf _{(\Delta \mathbb{C}, \Delta b \in \Omega)}\left\{\|\Delta \mathbb{C}, \Delta b\|_{F}\right\} .
$$

So $\Omega \neq \phi($ as $\Delta \mathbb{C}=0$ is a BCSCB matrix, $\Delta b=\widehat{\mathbb{C}} \widehat{x}-b)$. Hence,

$$
\xi^{2}(\widehat{x})=\inf _{(\Delta \mathbb{C}, \Delta b) \in \Omega}\left\{\|\Delta \mathbb{C}\|_{F}^{2}+\|\Delta \mathbb{C} \widehat{x}+\mathbb{C} \widehat{x}-b\|_{F}^{2}\right\} .
$$

Since

$$
\begin{gathered}
\|\Delta \mathbb{C}\|_{F}^{2}=m n \sum_{k=1}^{n} \sum_{l=1}^{m}\left(\varepsilon c_{k l}\right)^{2}, \\
\Delta \mathbb{C}=\mathbb{Q}\left(\sum_{k=1}^{n} \sum_{l=1}^{m} \varepsilon c_{k l} \Pi_{0}^{k-1} \otimes \Psi_{0}^{l-1}\right) \mathbb{Q}^{T},
\end{gathered}
$$

the question will be analysed in two different conditions.

(1) When $n$ is even,

$$
\begin{aligned}
& \|\Delta \mathbb{C} \hat{x}+\mathbb{C} \hat{x}-b\|_{F}^{2} \\
& =\mathbb{Q}\left(\begin{array}{cccc}
\varepsilon \Lambda_{11} & & & \\
& \ddots & & \\
& & \varepsilon \Lambda_{t t} & \\
& & & \varepsilon \Upsilon_{1}
\end{array}\right) \mathbb{Q}^{T} \hat{x}+\mathbb{C} \hat{x}-b \|_{F}^{2} \\
& =\left\|\left(\begin{array}{cccc}
\varepsilon \Lambda_{11} & & & \\
& \ddots & & \\
& & \varepsilon \Lambda_{t t} & \\
& & & \varepsilon \Upsilon_{1}
\end{array}\right)\left(\begin{array}{c}
x_{1}^{(0)} \\
\vdots \\
x_{t}^{(0)} \\
x_{t+1}^{(0)}
\end{array}\right)-r_{0}\right\|_{F}^{2} \\
& =\left\|\left(\begin{array}{c}
\left(\sum_{k=1}^{n} \sum_{l=1}^{m} \varepsilon c_{k l} \pi_{1}^{k-1} \otimes \Psi_{0}^{l-1}\right) x_{1}^{(0)} \\
\vdots \\
\left(\sum_{k=1}^{n} \sum_{l=1}^{m} \varepsilon c_{k l} \pi_{t}^{k-1} \otimes \Psi_{0}^{l-1}\right) x_{t}^{(0)} \\
\left(\sum_{k=1}^{n} \sum_{l=1}^{m} \varepsilon c_{k l} \operatorname{diag}\left(-\Psi_{0}^{l-1}, \Psi_{0}^{l-1}\right)\right) x_{t+1}^{(0)}
\end{array}\right)-r_{0}\right\|_{F}^{2}, \\
& =\left\|\Phi\left(\varepsilon c_{11}, \ldots, \varepsilon c_{1 m}, \ldots, \varepsilon c_{n 1}, \ldots, \varepsilon c_{n m}\right)^{T}-r_{0}\right\|_{F}^{2},
\end{aligned}
$$

where

$$
\begin{aligned}
& r_{0}=\mathbb{Q}^{T}(b-\mathbb{C} \widehat{x}), \\
& \mathbb{Q}^{T} \widehat{x}=\left(\begin{array}{llll}
x_{1}^{(0)} & \cdots & x_{t}^{(0)} & x_{t+1}^{(0)}
\end{array}\right)^{T}, \\
& \Phi=\left(\Phi_{1}, \Phi_{2}, \ldots, \Phi_{n-1}, \sum_{l=1}^{m} \operatorname{diag}\left(-\Psi_{0}^{l-1}, \Psi_{0}^{l-1}\right) x_{t+1}^{(0)}\right), \\
& \Phi_{k}=\left(\begin{array}{ccc}
\phi_{1, k, 1} & \cdots & \phi_{1, k, m} \\
\vdots & \ddots & \vdots \\
\phi_{t, k, 1} & \cdots & \phi_{t, k, m}
\end{array}\right) \text {, } \\
& \phi_{p, k, l}=\pi_{p}^{k-1} \otimes \Psi_{0}^{l-1} x_{p}^{(0)} \text {, } \\
& t=\frac{n}{2}-1, \quad p=1,2, \ldots, t, \\
& k=1,2, \ldots, n-1, \quad l=1,2, \ldots, m .
\end{aligned}
$$


(2) When $n$ is odd,

$$
\begin{aligned}
& \|\Delta \mathbb{C} \hat{x}+\mathbb{C} \hat{x}-b\|_{F}^{2} \\
& =\mathbb{Q}\left(\begin{array}{cccc}
\varepsilon \Lambda_{11} & & & \\
& \ddots & & \\
& & \varepsilon \Lambda_{t t} & \\
& & & \varepsilon \Upsilon_{2}
\end{array}\right) \mathbb{Q}^{T} \hat{x}+\mathbb{C} \hat{x}-b \|_{F}^{2} \\
& =\left\|\left(\begin{array}{cccc}
\varepsilon \Lambda_{11} & & & \\
& \ddots & & \\
& & \varepsilon \Lambda_{t t} & \\
& & & \varepsilon \Upsilon_{2}
\end{array}\right)\left(\begin{array}{c}
x_{1}^{(0)} \\
\vdots \\
x_{t}^{(0)} \\
x_{t+1}^{(0)}
\end{array}\right)-r_{0}\right\|_{F}^{2} \\
& =\left\|\left(\begin{array}{c}
\left(\sum_{k=1}^{n} \sum_{l=1}^{m} \varepsilon c_{k l} \pi_{1}^{k-1} \otimes \Psi_{0}^{l-1}\right) x_{1}^{(0)} \\
\vdots \\
\left(\sum_{k=1}^{n} \sum_{l=1}^{m} \varepsilon c_{k l} \pi_{t}^{k-1} \otimes \Psi_{0}^{l-1}\right) x_{t}^{(0)} \\
\left(\sum_{k=1}^{n} \sum_{l=1}^{n} \varepsilon c_{k l} \Psi_{0}^{l-1}\right) x_{t+1}^{(0)}
\end{array}\right)\right\|_{0}^{2} \\
& =\left\|\Phi\left(\varepsilon c_{11}, \ldots, \varepsilon c_{1 m}, \ldots, \varepsilon c_{n 1}, \ldots, \varepsilon c_{n m}\right)^{T}-r_{0}\right\|_{F}^{2},
\end{aligned}
$$

where

$$
\begin{aligned}
& r_{0}=\mathbb{Q}^{T}(b-\mathbb{C} \hat{x}) \\
& \mathbb{Q}^{T} \widehat{x}=\left(\begin{array}{llll}
x_{1}^{(0)} & \cdots & x_{t}^{(0)} & x_{t+1}^{(0)}
\end{array}\right)^{T}, \\
& \Phi=\left(\Phi_{1}, \Phi_{2}, \ldots, \Phi_{n-1}, \sum_{l=1}^{m} \Psi_{0}^{l-1} x_{t+1}^{(0)}\right), \\
& \Phi_{k}=\left(\begin{array}{ccc}
\phi_{1, k, 1} & \cdots & \phi_{1, k, m} \\
\vdots & \ddots & \vdots \\
\phi_{t, k, 1} & \cdots & \phi_{t, k, m}
\end{array}\right) \text {, } \\
& \phi_{p, k, l}=\pi_{p}^{k-1} \otimes \Psi_{0}^{l-1} x_{p}^{(0)}, \\
& t=\frac{n-1}{2}, \quad p=1,2, \ldots, t, \\
& k=1,2, \ldots, n-1, \quad l=1,2, \ldots, m .
\end{aligned}
$$

Let

$$
g\left(\varepsilon c_{11}, \ldots, \varepsilon c_{n m}\right)=m n \sum_{k=1}^{n} \sum_{l=1}^{m}\left(\varepsilon c_{k l}\right)^{2}+\left\|\Phi\left(\begin{array}{c}
\varepsilon c_{11} \\
\vdots \\
\varepsilon c_{n m}
\end{array}\right)-r_{0}\right\|_{F}^{2}
$$

and then

$$
\frac{\partial g}{\partial \varepsilon c_{k l}}=0
$$

which is equal to

$$
\begin{gathered}
\left(2 m n I_{m n}+2 \Phi^{T} \Phi\right)\left(\begin{array}{c}
\varepsilon c_{11} \\
\vdots \\
\varepsilon c_{n m}
\end{array}\right)-2 \Phi^{T} r_{0}=0, \\
\frac{\partial^{2} g}{\partial\left(\varepsilon c_{k l}\right)^{2}}=2 m n I_{m n}+2 \Phi^{T} \Phi>0 .
\end{gathered}
$$

As $g$ is a convex function of $\left(\varepsilon c_{11}, \ldots, \varepsilon c_{n m}\right)$, the point of the minimal value is

$$
\left(\begin{array}{c}
\varepsilon c_{11} \\
\vdots \\
\varepsilon c_{n m}
\end{array}\right)=\left(m n I_{m n}+\Phi^{T} \Phi\right)^{-1} \Phi^{T} r_{0}
$$

Substituting it back into (49), we obtain the following.

Theorem 6. Consider

$$
\begin{aligned}
\xi^{2}(x) & =m n r_{0}^{T} \Phi\left(m n I_{m n}+\Phi^{T} \Phi\right)^{-2} \Phi^{T} r_{0} \\
& =\left\|\left[\Phi\left(m n I_{m n}+\Phi^{T} \Phi\right)^{-1} \Phi^{T}-I_{m n}\right] r_{0}\right\|_{F}^{2}
\end{aligned}
$$

Let $\Phi=U \Sigma V^{*}$ be the singular value decomposition of $\Phi$, where $U$ and $V$ are unitary matrices, $\Sigma=\operatorname{diag}\left(\sigma_{1}^{\prime}, \ldots, \sigma_{n m}^{\prime}\right)$, and $\sigma_{j}^{\prime} \geq 0(j=1,2, \ldots, n m)$; then

$$
\begin{aligned}
\xi^{2}(x)= & m n r_{0}^{T} U \Sigma V^{T}\left(m n I_{m n}+\Sigma^{2}\right)^{-2} V \Sigma U^{T} r_{0} \\
& +\left\|\left[U \Sigma V^{T}\left(m n I_{m n}+\Sigma^{2}\right)^{-1} V \Sigma U^{T}-I_{m n}\right] r_{0}\right\|_{F}^{2} \\
= & m n r_{1}^{T} \Sigma\left(m n I_{m n}+\Sigma^{2}\right)^{-2} \Sigma r_{1} \\
& +\left\|\left[\Sigma\left(m n I_{m n}+\Sigma^{2}\right)^{-1} \Sigma-I_{m n}\right] r_{0}\right\|_{F}^{2} \\
= & m n r_{1}^{T} \Sigma\left(m n I_{m n}+\Sigma^{2}\right)^{-2} \Sigma r_{1} \\
& +\left\|\left[\Sigma\left(m n I_{m n}+\Sigma^{2}\right)^{-1} \Sigma-I_{m n}\right] r_{1}\right\|_{F}^{2} \\
= & m n r_{1}^{T} \Sigma\left(m n I_{m n}+\Sigma^{2}\right)^{-2} \Sigma r_{1} \\
& +m^{2} n^{2} r_{1}^{T}\left(m n I_{m n}+\Sigma^{2}\right)^{-2} r_{1} \\
= & r_{1}^{T}\left(\begin{array}{c}
\alpha_{1} \\
\ddots
\end{array}\right) r_{1},
\end{aligned}
$$

where $r_{1}=U^{T} r_{0}, \alpha_{j}=\left(m n \sigma_{j}^{\prime 2}+m^{2} n^{2}\right) /\left(m n+\sigma_{j}^{\prime 2}\right)^{2}=$ $m n /\left(m n+\sigma_{j}^{\prime 2}\right), j=1,2, \ldots, m n$.

Remark 7. As $\sigma_{j}^{2} \leq\|\Phi\|_{F}^{2}=m n\|\widehat{x}\|_{2}^{2}$, then $1+\|\widehat{x}\|_{2}^{2} \geq 1+$ $\sigma_{j}^{\prime 2} / m n$ can be obtained; hence, $m n /\left(m n+\sigma_{j}^{\prime 2}\right) \geq 1 /\left(1+\|\widehat{x}\|_{2}^{2}\right)$. 
From what we analysed above, the following algorithm can be obtained.

Algorithm 8. We have the following steps.

Step 1. Form the style spectral decomposition of the matrixes $\Pi$ and $\Psi$ :

$$
\Pi=Q \Pi_{0} Q^{T}, \quad \Psi=J \Psi_{0} J^{T} .
$$

Step 2. Form the block style spectral decomposition of the BCSCB matrix.

Step 3. Compute $r=b-\mathbb{C} \widehat{x}$.

Step 4. Compute $r_{0}=\mathbb{Q}^{T} r$.

Step 5. Compute

$$
\mathbb{Q}^{T} \widehat{x}=\left(\begin{array}{c}
x_{1}^{(0)} \\
\vdots \\
x_{t}^{(0)} \\
x_{t+1}^{(0)}
\end{array}\right)
$$

Step 6. Form $\Phi$.

Step 7. Compute the singular value decomposition of $\Phi$.

Step 8. Compute $\xi^{2}(\widehat{x})$.

\section{Numerical Example}

In this section, a simple numerical example is given to verify the conclusion above. Suppose that $n=2, m=3$ in the following example.

If the coefficient matrix of the BCSCB linear system is $\mathbb{C}=\operatorname{BCSCB}(3,5,2,7,9,8)$ and the constant vector $b=$ $(11,8,10,-8,9,3)^{T}$, now, three perturbations are given as follows:

$$
\begin{aligned}
\Delta \mathbb{C}_{1} & =0.01 \operatorname{BCSCB}(3,5,2,7,9,8), \\
\Delta b_{1} & =0.01(1,3,2,3,1,5)^{T}, \\
\Delta \mathbb{C}_{2} & =\operatorname{BCSCB}(0.01,0.02,0,0.015,0.033,0.01), \\
\Delta b_{2} & =(0.012,0.03,0.02,0.015,0.01,0.021)^{T}, \\
\Delta \mathbb{C}_{3} & =\operatorname{BCSCB}(0.01,0.02,0.015,0.01,0,0.01), \\
\Delta b_{3} & =(0.012,0.035,0.02,0.01,0.021,0.015)^{T} .
\end{aligned}
$$

From the equation $\widehat{\mathbb{C}} \widehat{x}=\widehat{b}$, where $\widehat{\mathbb{C}}, \widehat{b}$ are defined as above, the approximate solution of $\mathbb{C} x=b$ can be obtained as follows:

$$
x=\left(\begin{array}{c}
-1.0556 \\
0.3472 \\
-0.9306 \\
0.4444 \\
-0.1528 \\
1.5694
\end{array}\right), \quad \widehat{x}_{1}=\left(\begin{array}{c}
-1.0556 \\
0.3472 \\
-0.9306 \\
0.4444 \\
-0.1528 \\
1.5694
\end{array}\right)
$$

TABLE 1: The related date of the algorithm.

\begin{tabular}{lcccc}
\hline & $\epsilon$ & $\kappa$ & $\xi_{1}(\widehat{x})$ & $\xi_{2}(\widehat{x})$ \\
\hline Case 0 & 0 & 4.0000 & 0 & 0 \\
Case 1 & 0 & 4.0000 & $2.2112 e^{-15}$ & 0.3796 \\
Case 2 & $1.9396 e^{-4}$ & 4.0024 & 0.0028 & 0.1170 \\
Case 3 & 0.0028 & 3.9971 & 0.0202 & 0.0899 \\
\hline
\end{tabular}

$$
\widehat{x}_{2}=\left(\begin{array}{c}
-1.0553 \\
0.3472 \\
-0.9303 \\
0.4444 \\
-0.1528 \\
1.5694
\end{array}\right), \quad \widehat{x}_{3}=\left(\begin{array}{c}
-1.0531 \\
0.3439 \\
-0.9297 \\
0.4418 \\
-0.1492 \\
1.5699
\end{array}\right)
$$

where $x$ is the solution of $\mathbb{C} x=b$ and $\widehat{x}_{i}, i=1,2,3$, is the solution of $\left(\mathbb{C}+\Delta \mathbb{C}_{i}\right) x=b+\Delta b_{i}, i=1,2,3$, respectively.

Based on Algorithm 8, we obtain Table 1, where $\epsilon$ is the relative error of the BCSCB linear system, $\kappa=$ $\max \left\{\sigma_{i j}\right\} / \min \left\{\sigma_{i j}\right\}$ is the condition number, $\xi_{1}(\widehat{x})=\| b-$ $\mathbb{C} \hat{x} \|_{2} / \sqrt{1+\|\widehat{x}\|_{2}^{2}}$, and $\xi_{2}(\widehat{x})$ can be obtained from the algorithm.

From the numerical example, the accuracy of the conclusion and the effectiveness of the algorithm are verified.

\section{Conclusion}

In this paper, we consider the problems associated with the BCSCB matrix. The BCSCB matrix is an extension of the circulant matrix and skew circulant matrix. We give the form of the BCSCB matrix and obtain its block style spectral decomposition. The algorithm of the optimal backward perturbation is given. Furthermore, by circulant matrices technology, we will develop solving problems in [15-17].

\section{Conflict of Interests}

The authors declare that there is no conflict of interests regarding the publication of this paper.

\section{Acknowledgments}

This research was supported by the Development Project of Science \& Technology of Shandong Province (Grant no. 2012GGX10115) and the AMEP of Linyi University, China.

\section{References}

[1] T. N. Khokhlova and M. M. Kipnis, "The breaking of a delayed ring neural network contributes to stability: the rule and exceptions," Neural Networks, vol. 48, pp. 148-152, 2013.

[2] M. Bašić, "Characterization of quantum circulant networks having perfect state transfer," Quantum Information Processing, vol. 12, no. 1, pp. 345-364, 2013. 
[3] P. Frasca, R. Carli, F. Fagnani, and S. Zampieri, "Average consensus on networks with quantized communication," International Journal of Robust and Nonlinear Control, vol. 19, no. 16, pp. 17871816, 2009.

[4] V. Puttagunta and K. Kalpakis, "Accuracy vs. lifetime: linear sketches for aggregate queries in sensor networks," Algorithmica, vol. 49, no. 4, pp. 357-385, 2007.

[5] R. Happel, R. Hecht, and P. F. Stadler, "Autocatalytic networks with translation," Bulletin of Mathematical Biology, vol. 58, no. 5, pp. 877-905, 1996.

[6] D. Gómez, J. Gutierrez, and Á. Ibeas, "Optimal routing in double loop networks," Theoretical Computer Science, vol. 381, no. 1-3, pp. 68-85, 2007.

[7] J. L. Rigal and J. Gaches, "On the compatibility of a given solution with the data of a linear system," Journal of the Association for Computing Machinery, vol. 14, pp. 543-548, 1967.

[8] J. Li, Z. Jiang, N. Shen, and J. Zhou, "On optimal backward perturbation analysis for the linear system with skew circulant coefficient matrix," Computational and Mathematical Methods in Medicine, vol. 2013, Article ID 707381, 7 pages, 2013.

[9] X. Liu and X. Guo, "On optimal backward perturbation analysis for the linear system with block cyclic coefficient matrix," Numerical Mathematics, vol. 12, no. 2, pp. 162-172, 2003.

[10] J.-G. Sun and Z. Sun, "Optimal backward perturbation bounds for underdetermined systems," SIAM Journal on Matrix Analysis and Applications, vol. 18, no. 2, pp. 393-402, 1997.

[11] Z. Jiang, J. Li, and J. Zhou, "Optimal backward perturbation analysis for the block skew circulant linear systems with skew circulant blocks," Abstract and Applied Analysis, vol. 2014, Article ID 523102, 8 pages, 2014.

[12] Z. L. Jiang and Z. X. Zhou, Circulant Matrix, Chengdu Technology University, Chengdu, China, 1999.

[13] Z. L. Jiang and Z. X. Zhou, "Nonsingularity on level-2( $\left.\mathrm{r}_{1}, \mathrm{r}_{2}\right)$ circulant matrices of type $(\mathrm{m}, \mathrm{n})$," Chinese Quarterly Journal of Mathematics, vol. 11, no. 2, pp. 106-110, 1996.

[14] R. A. Horn and C. R. Johnson, Matrix Analysis, Posts and Telecom Press, 2005.

[15] H. Dong, Z. Wang, and H. Gao, "Distributed $H_{\infty}$ filtering for a class of markovian jump nonlinear time-delay systems over lossy sensor networks," IEEE Transactions on Industrial Electronics, vol. 60, no. 10, pp. 4665-4672, 2013.

[16] Z. Wang, H. Dong, B. Shen, and H. Gao, "Finite-horizon $H_{\infty}$ filtering with missing measurements and quantization effects," IEEE Transactions on Automatic Control, vol. 58, no. 7, pp. 17071718, 2013.

[17] D. Ding, Z. Wang, J. Hu, and H. Shu, "Dissipative control for state-saturated discrete time-varying systems with randomly occurring nonlinearities and missing measurements," International Journal of Control, vol. 86, no. 4, pp. 674-688, 2013. 


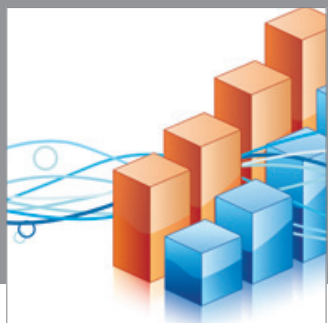

Advances in

Operations Research

mansans

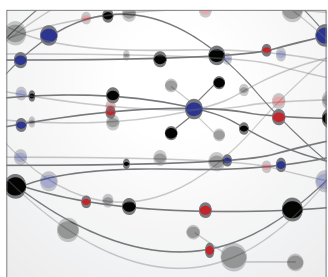

The Scientific World Journal
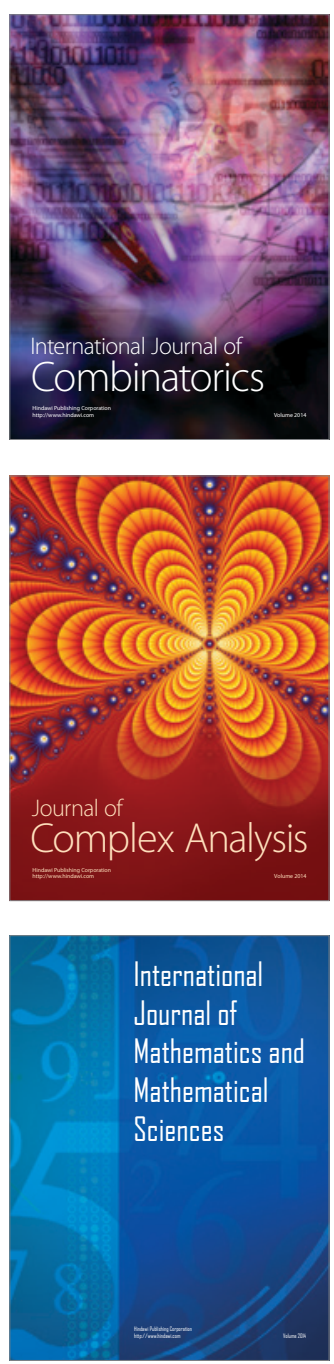
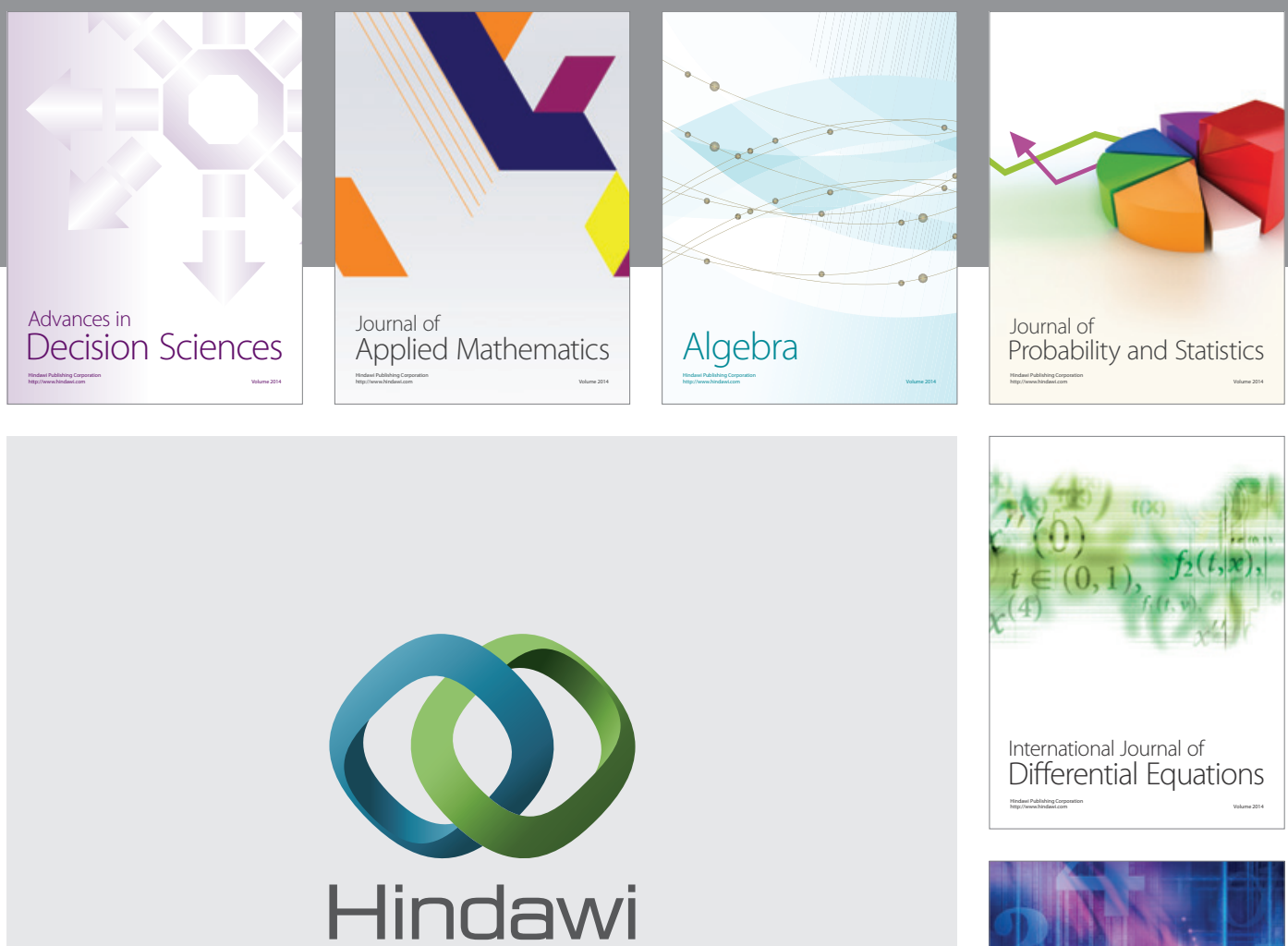

Submit your manuscripts at http://www.hindawi.com
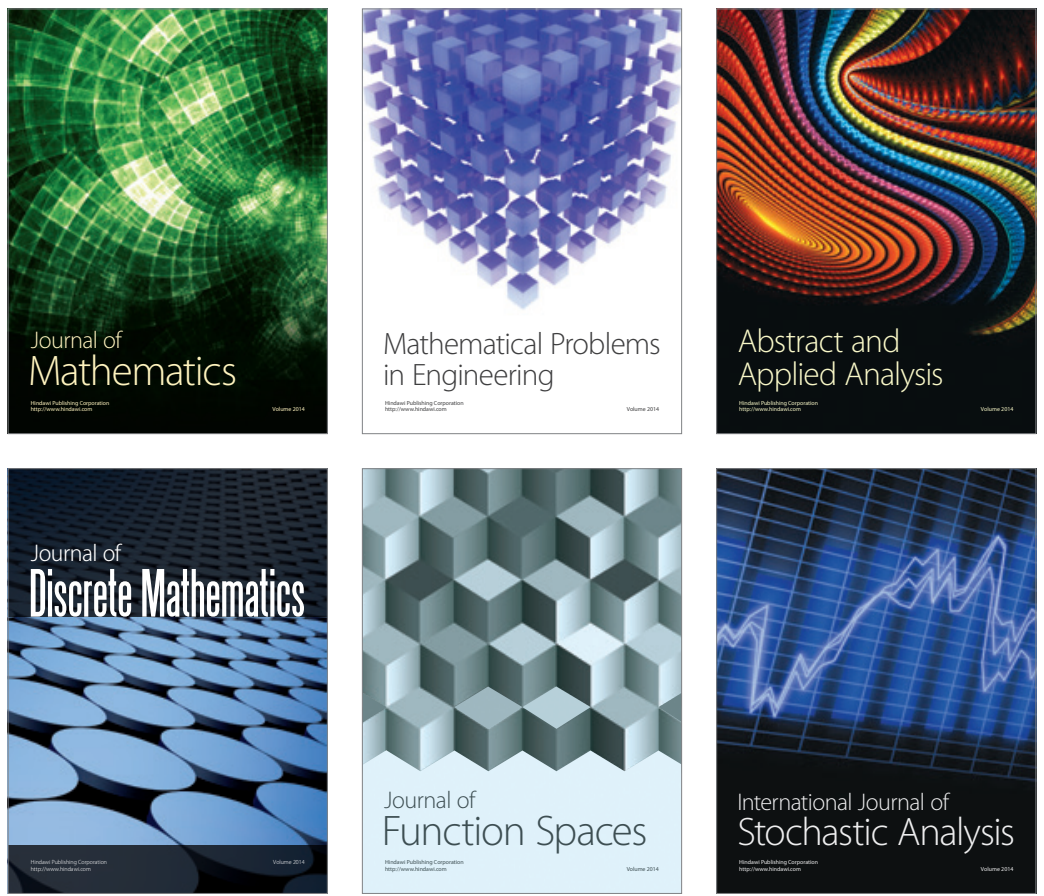

Journal of

Function Spaces

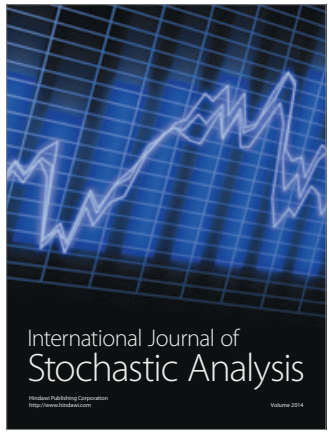

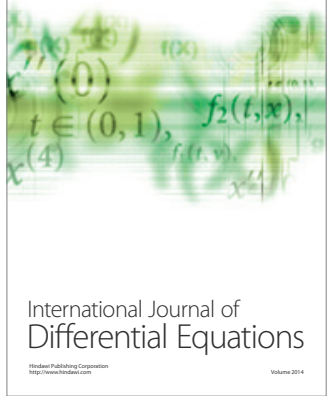
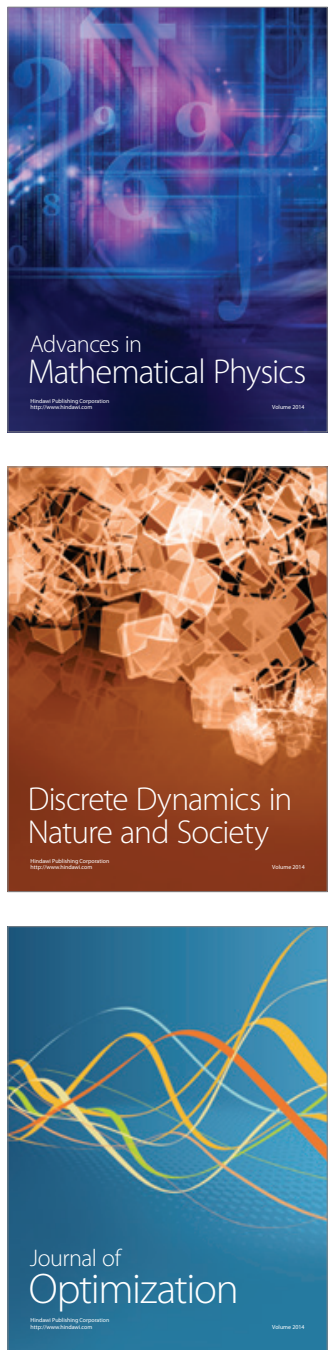